\title{
EMI Prediction of Slew-Rate Controlled I/O Buffers by Full-Wave and Circuit Co-Simulation
}

\author{
Namkyoung Kim, Jisoo Hwang, and SoYoung Kim
}

\begin{abstract}
In this paper, a modeling and co-simulation methodology is proposed to predict the radiated electromagnetic interference (EMI) from on-chip switching I/O buffers. The output waveforms of I/O buffers are simulated including the on-chip $\mathrm{I} / \mathrm{O}$ buffer circuit and the RC extracted on-chip interconnect netlist, package, and printed circuit board (PCB). In order to accurately estimate the EMI, a full-wave 3D simulation is performed including the measurement environment. The simulation results are compared with near-field electromagnetic scan results and farfield measurements from an anechoic chamber, and the sources of emission peaks were analyzed. For accurate far-field EMI simulation, PCB power trace models considering IC switching current paths and external power cable models must be considered for accurate EMI prediction. With the proposed EMI simulation model and flow, the electromagnetic compatibility can be tested even before the IC is fabricated.
\end{abstract}

Index Terms-I/O buffers, slew rate, EMI, near-field, far-field

\section{INTRODUCTION}

As the functions of processors become more complex and their operating frequencies become higher, electromagnetic interference (EMI) problems caused by

Manuscript received Apr. 3, 2014; accepted Jul. 26, 2014 Department of Semiconductor Systems Engineering, College of Information and Communication Engineering, Sungkyunkwan University, Suwon, Korea

E-mail : ksyoung@skku.edu data communication through the I/O buffers become more severe. To reduce the EMI from I/O buffers, the technique of adding on-chip decoupling capacitance on the power line, and optimizing the slew rate of $\mathrm{I} / \mathrm{O}$ buffers have been researched $[1,2]$. Trying to correct the EMI problems after the IC has been fabricated is too costly, so simulation based EMI estimation is necessary.

Integrated circuits(ICs) have often been considered to be the main noise source of EMI. The prediction of EMI generated from the ICs depends on both the on-chip and off-chip components. The on-chip simulations are done using a transistor level circuit simulator like SPICE and the EMI simulations are done using a 3D full-wave simulator. As a result, a methodology is needed to bridge the two tools for accurate EMI prediction considering the noise source, which is an IC, and the radiation path, which includes the package, PCB, cables, and the measurement environment such as an anechoic chamber. Co-simulation methods including on-chip and off-chip models can be useful in validating effective EMI reduction methods such as slew rate control. T. Sudo performed circuit simulations to find the time-domain waveform of the switching current and then performed EMI simulations considering the power distribution network (PDN) structure [1]. The radiated EMI emissions were reduced by controlling the slew rates of I/O buffers [2]. The far-field co-simulations have been applied to predict the EMI from a DC-DC converter in [3]. In [4] and [5], a full-wave simulation was done to extract the S-parameters, and the EMI prediction was done using a SPICE simulator. In [4], when EMI is predicted using the circuit model, the EMI can be estimated at one point, but the field distribution was not predicted. In [5], far-field EMI prediction was done using 


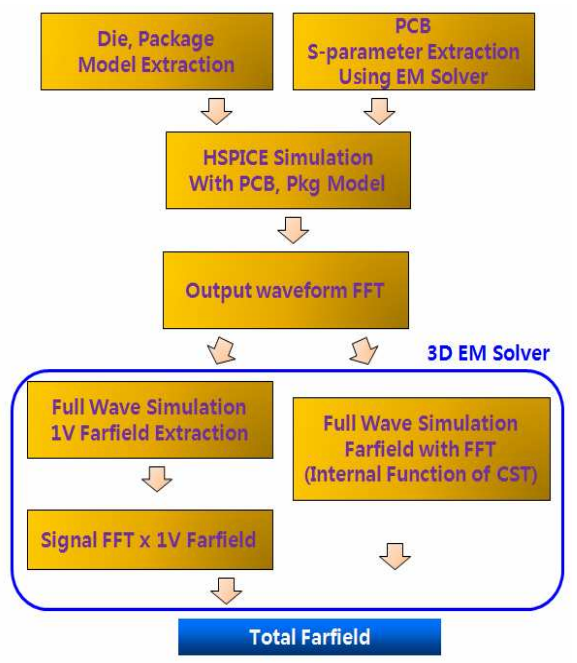

Fig. 1. Far-field co-simulation methodology.

the SPICE simulated IC output waveforms, but the differences between the simulation models and experiment were not fully investigated.

In this work, we show the on-chip and off-chip cosimulation methodology to predict EMI emissions and the results are compared with the near-field and far-field measurement results. We show that for switching I/O buffers, for accurate EMI prediction, we need to consider not only the PDN, but also the actual switching current path including the signal lines. By correlating the farfield simulation results, the effect of power cables on EMI resonances is identified. Using the EMI simulation platform constructed, methods to reduce the EMI from integrated circuits, such as controlling the signal slew rate and adding decoupling capacitance are examined.

\section{Co-Simulation Method}

The IC/package/PCB co-simulation was performed according to the flow shown in Fig. 1 [5]. HSPICE simulations were done using the post-layout RC extraction netlist of the IC, an RLC equivalent model of the package and the S-parameter of the PCB $[6,7]$. The frequency spectrum of the $\mathrm{I} / \mathrm{O}$ output waveform is extracted from the fast Fourier transform (FFT) of the time-domain output voltage waveform. Using a full-wave field solver, $1 \operatorname{volt}(\mathrm{V})$ source based far-field level is calculated including the 3D structure of the package and PCB. Then the far-field of active device under test(DUT) can be predicted through multiplying $1 \mathrm{~V}$ source based

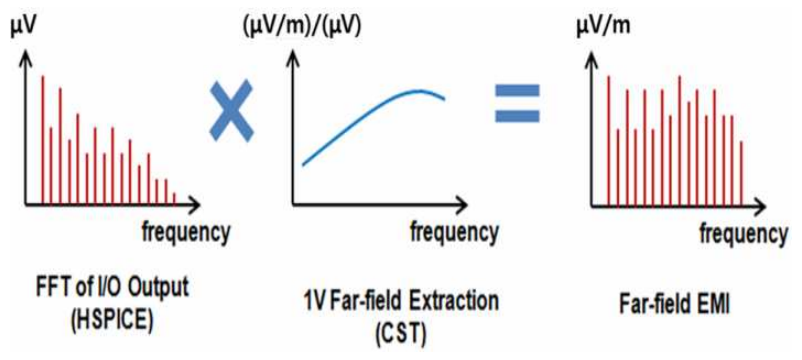

Fig. 2. Method to obtain frequency spectrum.
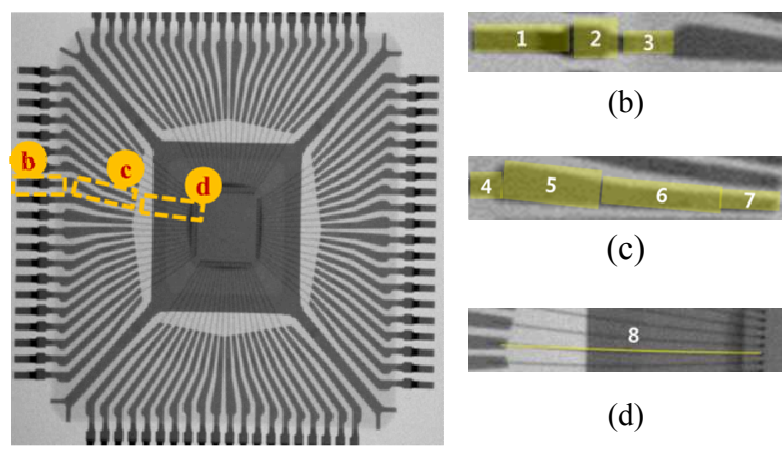

(c)

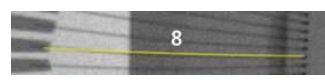

(a)

(d)

Fig. 3. Package parasitic component modeling (a) IC X-ray picture, (b) Pin, (c) Lead frame, (d) Wire bonding.

the far-field and the spectrum data of the $\mathrm{I} / \mathrm{O}$ buffer output signal as shown in Fig. 2.

\section{Simulation AND Measurement SETUP}

The structures of the PCB and the IC that were used in this paper are as follows. The slew rate of the I/O buffers can be adjusted. The size of the four-layer PCB is 10.4 $\mathrm{cm} * 10.4 \mathrm{~cm} * 1.0 \mathrm{~mm}$. The S-parameters are extracted from the PCB by using 2.5D MoM(Method of Moment) based simulator, SIWave, and the parasitic RLC components are extracted from the die by using a postlayout simulation [8]. Also the package shown in Fig. 3 is modeled as an series of RLC equivalent circuit $\pi$ model segments and their values are shown in Table 1. The circuit values are calculated from the following formula [9], where $t$ is the thickness, $w$ is the width, $h$ is the height and $l$ is the interconnect length, respectively.

$$
\begin{aligned}
& R=\frac{\rho \cdot l}{w \cdot t}, L=\frac{\mu_{0} l}{2 \pi}\left[\ln \left(\frac{8 h}{w+t}+1\right)\right] \\
& C=\varepsilon_{0} \varepsilon_{r}\left[1.13\left(\frac{w}{h}\right)+1.44\left(\frac{w}{w}\right)^{0.11}+1.46\left(\frac{t}{h}\right)^{0.42}\right]
\end{aligned}
$$


Table 1. Modeled parasitic component with respect to each segment in Fig. 3

\begin{tabular}{|c|c|c|c|}
\hline & $\mathrm{R}(\mathrm{m} \Omega)$ & $\mathrm{L}(\mathrm{nH})$ & $\mathrm{C}(\mathrm{fF})$ \\
\hline 1 & 0.731 & 0.26 & 191 \\
\hline 2 & 0.161 & 0.146 & 8.07 \\
\hline 3 & 0.356 & 0.213 & 9.13 \\
\hline 4 & 0.101 & 0.127 & 30.7 \\
\hline 5 & 0.431 & 0.396 & 78.2 \\
\hline 6 & 0.411 & 0.307 & 54.7 \\
\hline 7 & 0.388 & 0.259 & 43.8 \\
\hline 8 & 21.8 & 1.24 & 132 \\
\hline
\end{tabular}

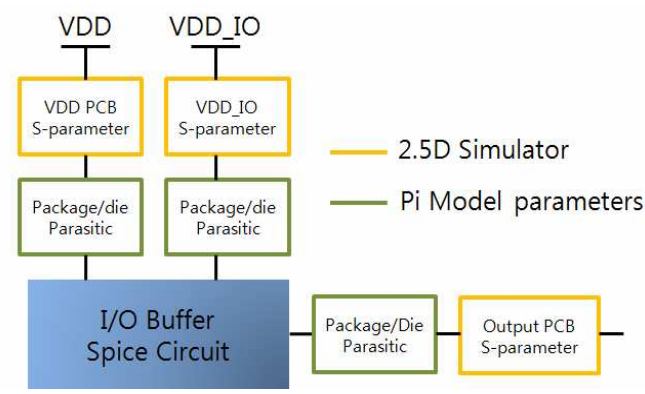

Fig. 4. I/O buffer circuit simulation configuration using HSPICE.

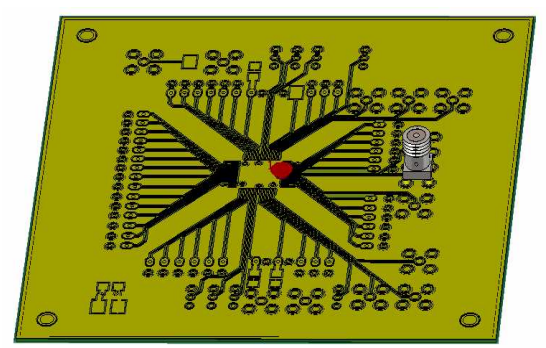

Fig. 5. PCB simulation modeling using CST.

Fig. 4 describes the overall circuit simulation model for EMI prediction. It includes the circuit schematic, the parasitic $\mathrm{RC}$ components extracted from the die, a package model, the PCB models. With this model, the time domain output voltage waveform is obtained from SPICE, and FFT can be performed to get the frequency spectrum.

The $1 \mathrm{~V}$ source based far-field curve is extracted from the modeled PCB by the full-wave simulator as shown in Fig. 5 [10]. By multiplying the FFT of the I/O buffer output waveform which is the source of the radiated emission, the total far-field radiation can be estimated.

The experiment is implemented with the following conditions as shown in Fig. 6. A waveform generator injects $20 \mathrm{MHz}$ of $1.2 \mathrm{~V}$ pulse into the $\mathrm{I} / \mathrm{O}$ buffers in the PCB. The supply voltage is $1.2 \mathrm{~V}$. The output waveform

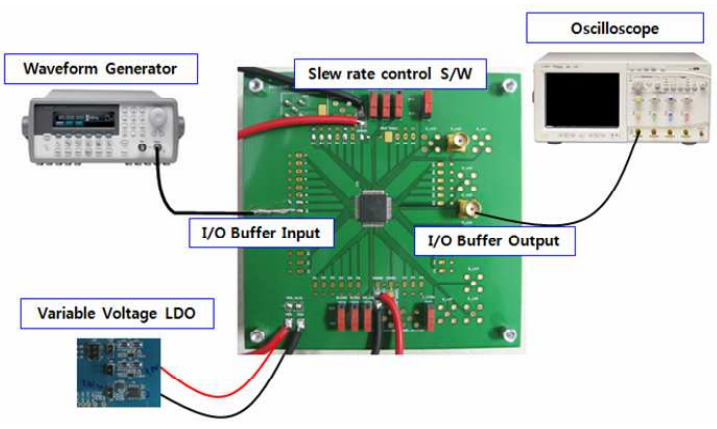

Fig. 6. Experiment setup.

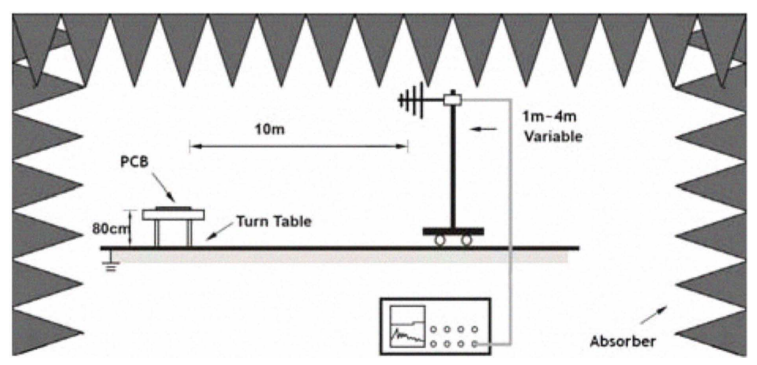

Fig. $7.10 \mathrm{~m}$ anechoic chamber.

is observed using the oscilloscope.

The far-field radiated emission (RE) level was measured in a $10 \mathrm{~m}$ chamber as shown in Fig. 7 [11, 12]. To reduce the noise of the power supply, the variable voltage LDO (Low Drop Out) is used. In the far-field condition, the noise from the I/O buffers cannot be detected because the signal is transferred well in the micro-strip lines, so the radiation from the signal line is extremely low. In order to measure the harmonic noise level, a $62 \mathrm{~mm}$ monopole antenna is attached to output termination of the signal line, which easily emits the I/O buffer signal to the air.

\section{EXPERIMENTAL RESULTS}

The near-field mapping system is used to measure the magnetic field level on the PCB, and a x-directional magnetic field probe is used. The near-field measurement results according to different slew rates are shown in Fig. 8. In Fig. 8, for a faster slew rate, the magnetic field of the buffer is stronger in the IC and PCB. The near-field simulation results using the $3 \mathrm{D}$ full-wave simulator CST are shown in Fig. 9. In the simulation, a simplified source model is used for IC. The near-field varies depending on the slew rate and the waveform of the I/O buffers. The 


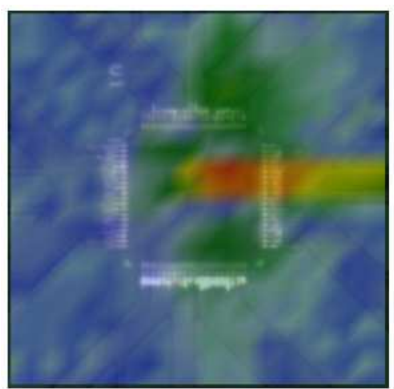

(a)

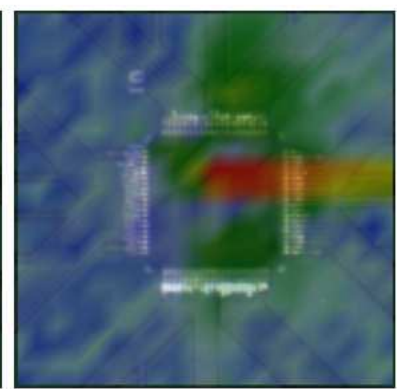

(b)
Fig. 8. Near-field EM scan measurement results at $800 \mathrm{MHz}$ (a) Minimum slew rate, (b) Maximum slew rate.

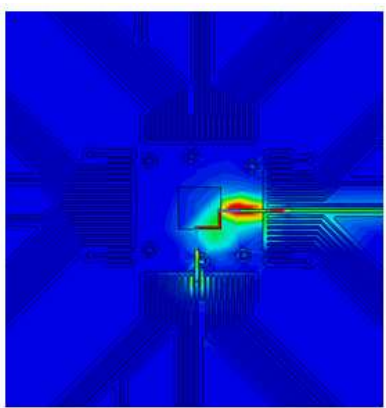

(a)

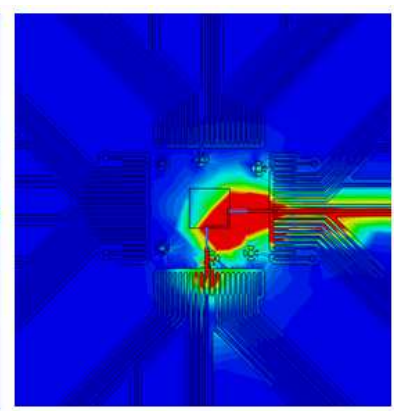

(b)
Fig. 9. Near-field simulation results at $800 \mathrm{MHz}$ (a) Minimum slew rate, (b) Maximum slew rate.

near-field measurement and simulation results show the maximum field intensity at the same location and show a similar trend as the slew rate changes.

According to the measurement method described in section II, the far-field measurement was done and the results are shown in Fig. 10. The overall magnitude of frequency spectrum is higher as the slew rate becomes faster.This shows that controlling the rise time of the switching buffers is a very effective way of solving EMI problem.

The far-field measurement shows the maximum peak near $850 \mathrm{MHz}$ due to the antenna attached at the I/O buffer output for radiated emission measurement in the chamber. The antenna attached to output is $62 \mathrm{~mm}$, and has a resonance near $850 \mathrm{MHz}$. Under the $300 \mathrm{MHz}$, the far-field measurement showed wide peak, showing that the noise radiated through $30 \mathrm{~cm}$ power cable is the dominant cause of EMI at low frequency. To see the effect of emission from the power cable, measurements were performed by varying the power cable length, and it is observed that the emissions near $300 \mathrm{MHz}$ are reduced when the power cable length was shortened as shown in Fig. 11.

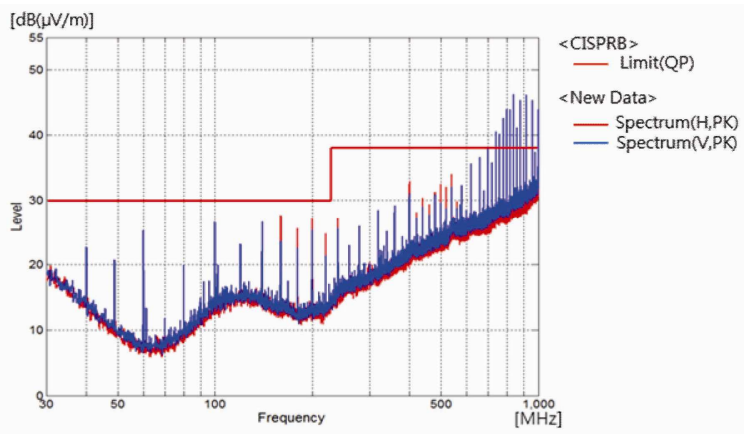

(a)

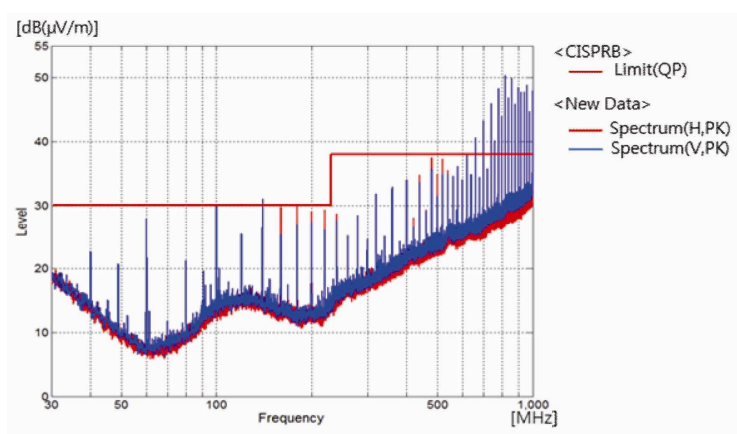

(b)

Fig. 10. Far-field measurement from anechoic chamber (a) Minimum slew rate, (b) Maximum slew rate.

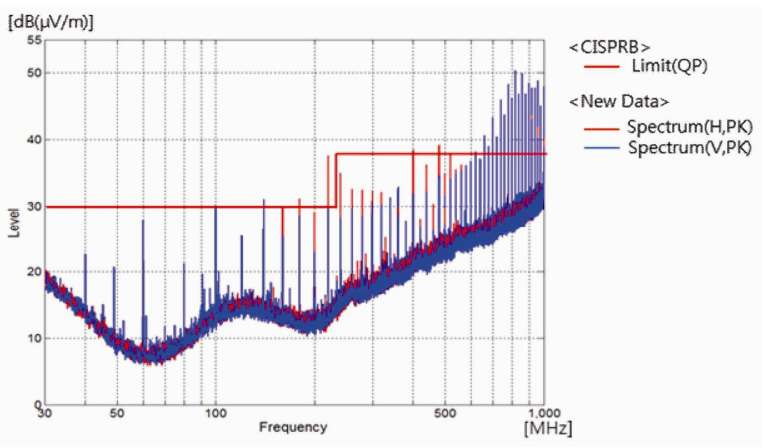

(a)

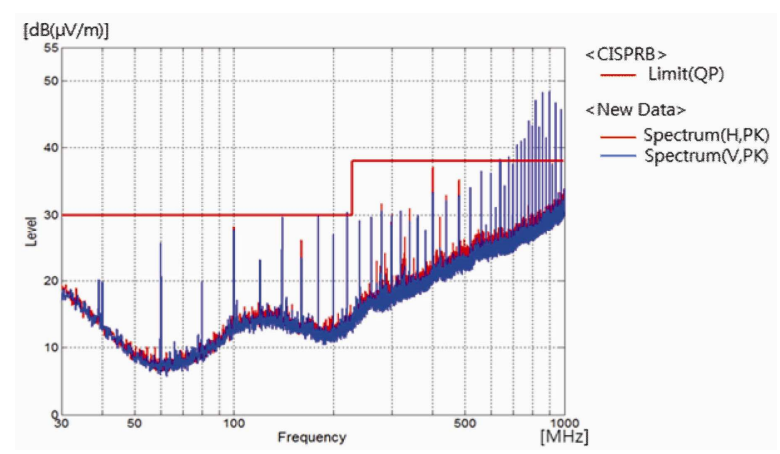

(b)

Fig. 11. Effect of battery wire length on measured far-field EMI (a) Long power cable $(30 \mathrm{~cm})$, (b) Short power cable $(3 \mathrm{~cm})$. 


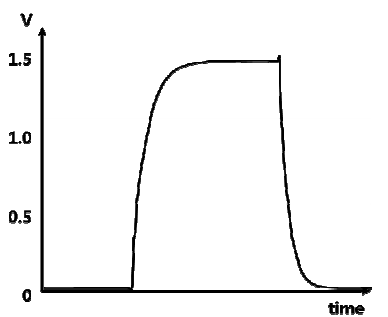

(a)

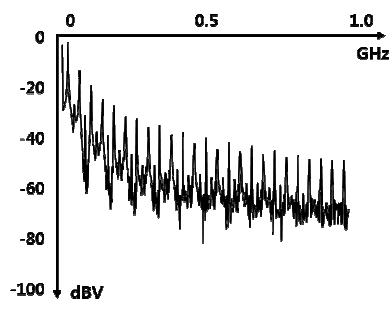

(b)
Fig. 12. SPICE simulation results of $I / O$ buffer output (a) Voltage waveform in time domain, (b) Voltage spectrum in frequency domain.

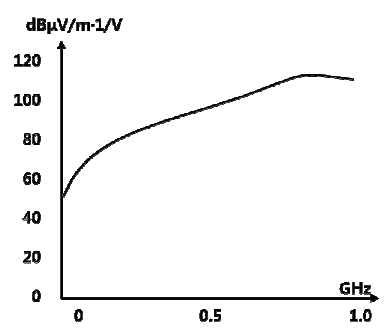

(a)

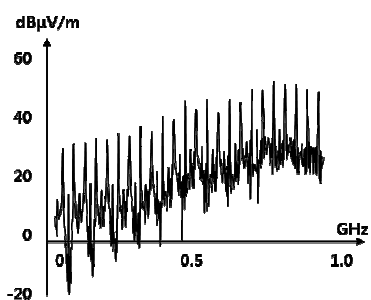

(b)
Fig. 13. Far-field simulation results (a) $1 \mathrm{~V}$ far-field curve, (b) Total far-field of I/O buffer.

The far-field simulation results are obtained through the proposed co-simulation methodology by multiplying the FFT of HSPICE-simulated output signal and the 3D full-wave-simulated $1 \mathrm{~V}$ far-field result. Output signal is obtained by SPICE simulation and its results are shown in Fig. 12. Figs. 12(a) and (b) show time domain voltage waveform and frequency domain voltage spectrum, respectively. Fig. 13(a) shows $1 \mathrm{~V}$ far-field curve which is obtained by 3D full-wave simulator. Finally, the total far-field result as shown in Fig. 13(b) is obtained by summing graphs in Figs. 12(b) and 13(a).

The switching current directions when the output of the on-chip I/O buffer rises and falls are shown in Fig. 14. When output clock rises, the power trace and the output trace are short during transition. The transition current flows from the power to output node. Due to this current, noise is radiated through the trace between power and output. When the output clock falls, the output trace and the ground are short, and discharging current flows to the ground. During this transition, noise is radiated through the trace between ground and output. In our test PCB structure, as the power trace is longer than that of ground trace, it is expected that the power trace has more

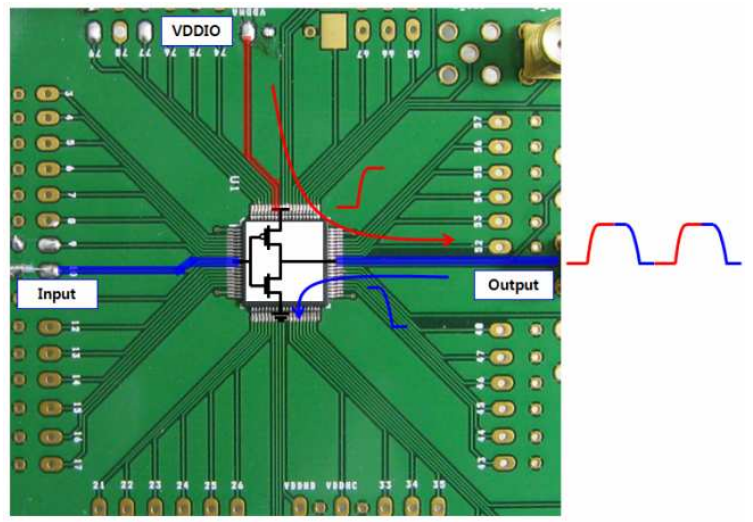

Fig. 14. The current flow in a switching I/O buffer.
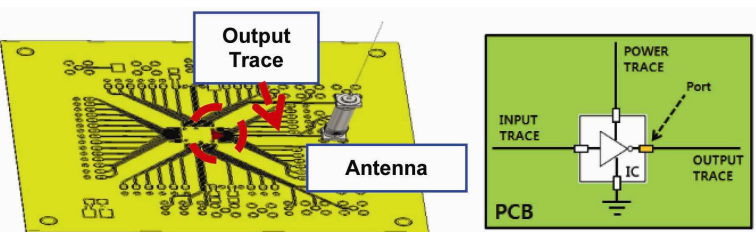

(a)
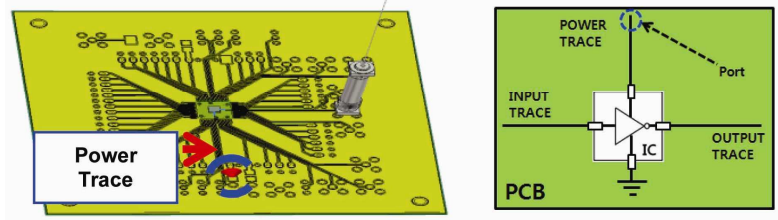

(b)

Fig. 15. Method of selecting port position (a) Original method, (b) Proposed method.

dominant effect on the electromagnetic radiation. In our initial EMI simulation setup using 3D EM solver, we placed the port of radiation at the output trace. But for correct prediction, the port needs to be designated to include the power trace and the output trace as shown in Fig. 15(b). Fig. 16 shows the far-field simulation results obtained by simulating the model shown in Fig. 15. The total simulated far-field and measurement results are compared. The far-field magnitude of modified model increases near $500 \mathrm{MHz}$ which is closer to the measured one. When the port position is located on the power trace considering current flow, more accurate far-field results can be achieved. But the measurement results show the weakness of the simulation method under $300 \mathrm{MHz}$. In the far-field simulation, power cable was not considered, so if the modeling of power cable is implemented, the accuracy of the simulation will improve. 


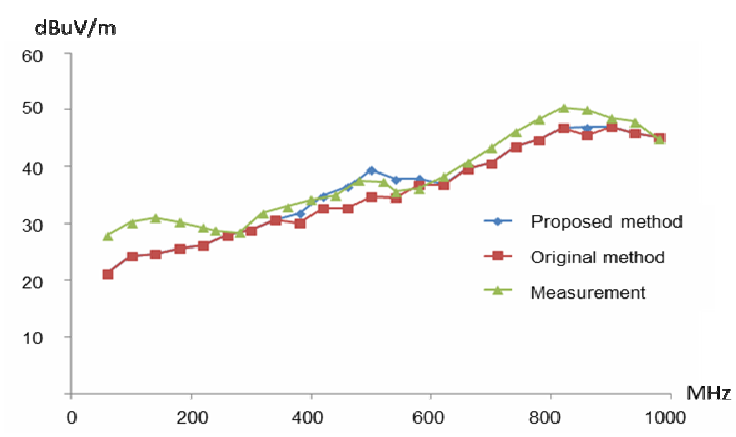

Fig. 16. Measurement and simulation far-field comparison.

\section{CONCLUSIONS}

In this study, an accurate far-field simulation methodology of $\mathrm{I} / \mathrm{O}$ buffers considering the slew rate is proposed. The waveforms of the I/O buffer output are simulated including circuit schematic and $\mathrm{RC}$ extracted on-chip interconnect netlist, package, and printed circuit board (PCB). The estimated output waveform is the source of the full-wave electromagnetic simulation. The simulation and measurement results match well when the port location is properly selected by considering the current through the PCB power line in the far-field simulation. The results also show that by controlling the slew rate of I/O buffers, the radiated EMI can be reduced. With the proposed EMI co-simulation flow, electromagnetic compatibility of other types of integrated circuits can be evaluated through the proposed co-simulation method. Moreover, the models and effectiveness of EMI reduction solutions at IC, package level can be evaluated through simulation before IC tape-out.

\section{ACKNOWLEDGMENTS}

This research was supported by the National Research Foundation of Korea(NRF) grant funded by the Korea government(MSIP) (No.NRF-2014R1A2A2A01006595). This work was supported by the IC Design Education Center (IDEC). Authors would like to thank Prof. Wansoo Nah of Sungkyunkwan University for discussions.

\section{REFERENCES}

[1] T. Sudo, et al, "Characterization of on-chip capacitance effects for $\mathrm{I} / \mathrm{O}$ circuits and core circuits,"IEEE 10th Topical Meeting on Electrical Performance of Electronic Packaging, pp.73-76, 2001.

[2] Wei-Da Guo,et al, “An On-/Off-Chip Co-Design Methodology for Suppressing Radiated Emissions from the High-Definition DTV System,'Topical Meeting on Advanced Research in EMC of ICs, IEEE Asia-Pacific Symposium on Electromagnetic Compatibility, pp.1064-1067, Apr. 2010.

[3] A. Bhargava, et al, "EMI prediction in switched power supplies by full-wave and non-linear circuit co-simulation,"IEEE International Symposium on Electromagnetic Compatibility, pp.44-46, Aug. 2009.

[4] SangKeun Kwak, Jeongmin Jo and SoYoung Kim,"Modeling and Co-Simulation of I/O Interconnects for On-Chip and Off-Chip EMI Prediction," IEEE Asia-Pacific Symposium on Electromagnetic Compatibility, pp.821-824, May, 2012.

[5] Namkyoung Kim, et al, "EMI prediction in slew ratecontrolled switching I/O buffers", IEEE AsiaPacific Symposium on Electromagnetic Compatibility, pp.181-184, May 2013

[6] HSPICE, Synopsys Inc

[7] Calibre, Mentor Graphics

[8] SIwave, Ansys Inc.

[9] N. Delorme, M. Belleville, and J. Chilo, "Inductance and Capacitance Analytic Formulas for VLSI Interconnects," Electronics Letters, 23rd, Vol.32, No.11, May 1996.

[10] CST Microwave Studio, Computer Simulation Technology

[11] CISPR 22, Information technology equipment Radio disturbance characteristics - Limits and methods of measurement, Edition 5.2, Mar., 2006.

[12] Cheng-Chang Chen, et al, "A Study of PCB EMI Measurement and Simulation,"IEEE Asia-Pacific Symposium on Electromagnetic Compatibility, pp.736-739, Apr. 2010.

[13] Michel Mardiguian, Controlling Radiated Emissions by Design, 2nd edition, Springer, 2001. 


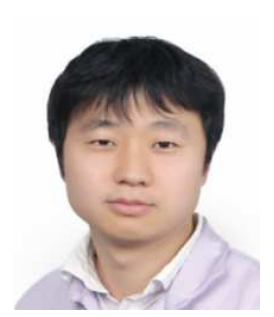

Namkyoung Kim received the B.S degree in Electric, Electronic and Radio Engineering from Korea University, Seoul, Korea, in 2004 and M.S. degree in Digital Media Communication Engineering from Sungkyunkwan University, Suwon,

Korea, in 2013. He is working in R\&D Solution Lab. in DMC R\&D Center of Samsung Electronics, from 2004. His research interests include signal and power integrity and EMI on IC level.

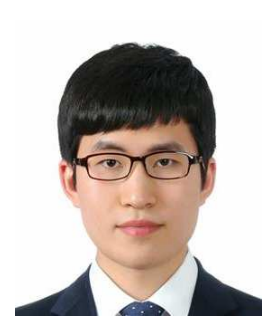

Jisoo Hwang received the B.S degree from the Department of Semiconductor Systems Engineering, Sungkyunkwan University, Suwon, Korea, in 2013. He is currently pursuing the M.S. degree with the College of Information and Communication Engineering, Sungkyunkwan University. His research interests include interconnect delay modeling and EMC/EMS.

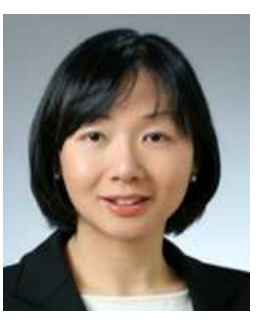

SoYoung Kim received a B.S. degree in Electrical Engineering from Seoul National University, Seoul, Korea in 1997 and M.S. and Ph.D. degrees in Electrical Engineering from Stanford University, Stanford, CA in 1999 and 2004, respectively. From 2004 to 2008, she was with Intel Corporation, Santa Clara, CA, and from 2008 to 2009, she was with Cadence Design Systems. She is currently an Associate Professor with the Department of Semiconductor Systems Engineering, College of Information and Communication Engineering, Sungkyunkwan University, Suwon, Korea. Her research interests include device and interconnect modeling, signal integrity, power integrity and electromagnetic interference in electronic systems. 